\title{
Opportunists in peritonitis in continuous ambulatory peritoneal dialysis: the example of Paracoccus yeei
}

\author{
Christelle Fosso $^{1}$, Evelyne Maillart ${ }^{2}$, Bram Beun ${ }^{3}$, Fahd Touzani ${ }^{1}$, Bhavna Mahadeb ${ }^{1}$, and \\ Philippe Clevenbergh ${ }^{2}$ \\ ${ }^{1} \mathrm{CHU}$ Brugmann \\ ${ }^{2}$ Brugmann University Hospital \\ ${ }^{3}$ CHU Saint-Pierre
}

January 8, 2021

\begin{abstract}
We report a case of $\mathrm{P}$. yeei CAPD-peritonitis in a patient experiencing repeated episodes of peritonitis. P. yeei, an emerging opportunistic pathogen, takes advantage of specific virulence factors and peculiar circumstances. We discuss the exogenous or endogenous microbial source of the peritonitis and guidelines to decrease its occurrence.
\end{abstract}

Opportunists in peritonitis in continuous ambulatory peritoneal dialysis: the example of

Paracoccus yeei

Fosso $\mathrm{C}^{1}$, Maillart $\mathrm{E}^{2}$, Beun $\mathrm{A}^{3}$, Touzani $\mathrm{F}^{1}$, Mahadeb $\mathrm{B}^{4}$, Clevenbergh $\mathrm{P}^{2}$

Corresponding author: Dr Clevenbergh Philippe: Infectious Diseases Department, CHU Brugmann, 4, Place A Van Gehuchten, 1020 Brussels Belgium, Philippe.CLEVENBERGH@chu-brugmann.be

Key Clinical Message

Peritonitis is a serious problem for patients undergoing peritoneal dialysis (PD) with incidence varying between centers. Paracoccus yee i, a soil microorganism with specific virulence factors, is an opportunistic pathogen. We report a case of CAPD -peritonitisdue to P. yeei. International guidelines exist to decrease exogenously and endogenously acquired CAPD-peritonitis.

Key words

Paracoccus yee i, peritonitis, peritoneal dialysis, complications, prevention.

Abstract

We report a case of $P$. yeei CAPD-peritonitis in a patient experiencing repeated episodes of peritonitis. $P$. yeei, an emerging opportunistic pathogen, takes advantage of specific virulence factors and peculiar circumstances. We discuss the exogenous or endogenous microbial source of the peritonitis and guidelines to decrease its occurrence.

Introduction

Serious complications of peritoneal dialysis are peritonitis, catheter exit site and tunnel infections. Over 0.5 episodes/patient-year rate of peritonitis has been reported in some facilities (1) with up to $5 \%$ mortality rate (2). In 10-20\%, no etiological organism can be detected. This rate can be decreased by direct inoculation of dialysate in blood-culture bottles (3) or amplification and identification of $16 \mathrm{~S}$ rRNA. Some peritonitis may 
be truly aseptic. Skin colonizers such asS. epidermidis and S. aureus, Gram-negative organisms or even fungus are involved owing to the mode of entry: skin and catheter-related, gut-associated translocation or hematogenous seeding. Various unexpected exogenous opportunistic micro-organisms originating from the patient's environment have been reported to cause exit site, tunnel infection and peritonitis $(4,5)$. Paracoccus yeei is an aerobic Gram-negative coccobacillus, found in soil and water. It can develop biofilms on plastic surfaces and has recently been identified as an opportunistic pathogen in human disease (6). Few cases of $P$. yeei -induced peritonitis in patients undergoing CAPD have been described $(7,8,9,10)$. We report such a case successfully treated with intraperitoneal amoxicillin in a patient experiencing several episodes of exogenous catheter infections.

Case report

This is the case of a 50-year-old woman. Her medical history reveals chronic depression, social isolation, linguistic barrier, and hypertension. She has been treated since December 2011 for end-stage renal disease of undetermined etiology. Starting by hemodialysis, she was transferred to PD in July 2012. In PD, she presented several tunnel infections and two episodes of peritonitis refractory to treatment requiring removal and reinsertion of PD catheter. In September 2018, she presented with diffuse abdominal pain with anorexia, diarrhea and shivering for 4 days although afebrile.

Laboratory analysis revealed: white blood cell count of $9.1910^{3} / \mu \mathrm{L}$ with normal differentiation and a Creactive protein level of $47.7 \mathrm{mg} / \mathrm{L}$ (normal value $<10 \mathrm{mg} / \mathrm{L}$ ). Peritoneal fluid analysis revealed a leukocyte count of $346 / \mu \mathrm{L}$, with $51 \%$ monocytes, $41 \%$ polymorphonuclear cells and $4 \%$ lymphocytes. The peritoneal catheter exit site was of normal appearance. Empirical treatment was initiated with intraperitoneal (IP) vancomycin $30 \mathrm{mg} / \mathrm{kg}$ and amikacin $2 \mathrm{mg} / \mathrm{kg}$ according to local protocol. Subsequent effluent dialysate analysis showed an initial rise in WBC count to $2111 / \mu \mathrm{L}$ (polymorphonuclear predominance of $49 \%$ ). Direct examination showed Gram-negative cocci, and culture yielded Paracoccus yeei. Drug susceptibility was tested for amoxicilline and amoxicilline-clavulanate, both of which were sensitive. Empiric treatment was discontinued on the second day of hospitalization and replaced by amoxicillin $150 \mathrm{mg}$ IP per liter of effluent. The total treatment duration was of 3 weeks with progressive decline and normalization of effluent cytology on the 9 th day following diagnosis. However, the patient developed other catheter infections with $S$. oralis and P. mirabilis involving the exit site, later P. putida and C. freundii peritonitis, and lastly a S. pneumoniae peritonitis in January 2020.

\section{Discussion:}

The genus Paracoccus comprises 17 species found in soil and water. Only P. yeei has been identified as a pathogen causing disease in humans as it possesses specific genes of virulence (6). Spectrum of disease includes CAPD-associated peritonitis and bacteremia associated with cellulitis, myocarditis in a hearttransplant, bacteremia in a cirrhotic patient, arthritis, and keratitis $(11,12,13,14,15)$. Previous reports have demonstrated antibiotic susceptibility to beta-lactams, fluoroquinolones, and aminoglycosides. Amoxicillin and other beta-lactams are likely effective treatment options for P. yeei. Our patient received IP amoxicillin for 3 weeks. IP administration of antibiotics is the preferred route to treat peritonitis for a duration of $2-3$ weeks according to the germ identified. IP dosing recommendations are available for many antibiotics (2). The incidence of $\mathrm{PD}$ peritonitis varies greatly according to the reporting centers and ranges from 0.2 to over 0.5 episodes per patient/year (1). Various interventions are recommended to prevent catheter and tunnel infections. Skin/catheter-related exogenous infections can be prevented by general hygiene, patient education and training in practicing home CAPD, Staphylococcus aureus decolonization, antibiotic ointment of the exit site, among others (16). Endogenous, bacterial translocation-associated peritonitis can be prevented by prevention of constipation, and antibio-prophylaxis prior to various invasive endoscopic procedures (17).

\section{Conclusion}

Environmental germs of low virulence can cause peritoneal dialysis catheter-associated peritonitis providing opportunistic circumstances. We report a case of $P$. yeei PD peritonitis successfully treated with intraperitoneal ampicillin in a patient experiencing several episodes of infections with various germs. $P$. yeei is an 
emerging opportunistic pathogen. Various prophylactic measures can be implemented to reduce the rate PD peritonitis from both exogenous and endogenous sources.

Acknowledgement statement

None

References:

1. Perl J, Fuller DS, Bieber BA, et al . Peritoneal Dialysis-Related Infection Rates and Outcomes: Results From the Peritoneal Dialysis Outcomes and Practice Patterns Study (PDOPPS). Am J Kidney Dis. 2020 ;76(1):42-53. doi: 10.1053/j.ajkd.2019.09.016

2. Salzer WL. Peritoneal dialysis-related peritonitis: challenges and solutions. Int J Nephrol Renovasc Dis. 2018; 11:173-186. doi: 10.2147/IJNRD.S123618.

3. Pindi G, Kawle V, Rao Sunkara R, Satyanarayana Darbha M, Garikaparthi S. Continuous ambulatory peritoneal dialysis peritonitis: Microbiology and outcomes. Indian J Med Microbiol. Jan-Mar 2020;38(1):72-77. doi: 10.4103/ijmm.IJMM_20_251

4. Mu H, Yang M, Zhang Y, Zhang Y, Wang J, Yuan W, Rong S. Pet-related Pasteurella multocida induced peritonitis in peritoneal dialysis: a case report and review of the literatures. BMC Nephrol. 2020 Mar 19;21(1):102. doi: 10.1186/s12882-020-01765-1.

5. Inoue $\mathrm{H}$, Washida $\mathrm{N}$, Morimoto $\mathrm{K}$, et al . Non-Tuberculous Mycobacterial Infections Related to Peritoneal Dialysis.Perit Dial Int. Mar-Apr 2018;38(2):147-149. doi: 10.3747/pdi.2017.00172. DOI: 10.3747/pdi.2017.00172

6. Lasek R, Szuplewska M, Mitura M, et al . Genome Structure of the Opportunistic Pathogen Paracoccus yeei (Alphaproteobacteria) and Identification of Putative Virulence Factors. Front Microbiol. 2018 Oct 25;9:2553. doi: 10.3389/fmicb.2018.02553. eCollection 2018.

7. Wallet F, Blondiaux N, Foy CL, Loïez C, Armand S, Pagniez D, Courcol RJ. Paracoccus yeei: a new unusual opportunistic bacterium in ambulatory peritoneal dialysis. Int J Infect Dis. 2010;14(2):e173-4. doi: 10.1016/j.ijid.2009.03.030.

8. Sastre A, González-Arregoces J, Romainoik I, Mariño S, Lucas C, Monfá E, Stefan G, de León B, Prieto M. Paracoccus yeei peritonitis in peritoneal dialysis. Nefrologia ;36(4):445-6. doi: 10.1016/j.nefro.2016.02.009

9. Arias MA, Clark J. Paracoccus yeei as a cause of peritoneal dialysis peritonitis in the United Kingdom. IDCases. 2019;15:e00486. doi: 10.1016/j.idcr.2019.e00486

10. Nakayama T, Morimoto K, Uchiyama K. Peritoneal dialysis-related peritonitis caused by Paracoccus yeei. Ther Apher Dial. 2020 Nov 1. doi: 10.1111/1744-9987.13604.

11. Funke G, Frodl R, Sommer H. First Comprehensively Documented Case of Paracoccus yeei Infection in a Human. J Clin Microbiol. 2004; 42(7): 3366-3368.

12. Schweiger M, Stiegler P, Scarpatetti M et al. Case of Paracoccus yeei infection documented in a transplanted heart. Transpl Infect Dis. 2011;13(2):200-3.

13. Sack J, Peaper DR, Mistry $P$ et al. Clinical implications of Paracoccus yeeii bacteremia in a patient with decompensated cirrhosis. Malinis MIDCases. 2016; 7:9-10.

14. Coiffier G, Gougeon A, Albert JD, Le Bars H. Arthritis due to Paracoccus yeei]. Med Mal Infect. 2013;43(6):254-5. doi: 10.1016/j.medmal.2013.04.004.

15. Kanis MJ, Oosterheert JJ, Lin S, Boel CH, Ekkelenkamp MB. Corneal graft rejection complicated by Paracoccus yeei infection in a patient who had undergone a penetrating keratoplasty. J Clin Microbiol. 2010;48(1):323-5. doi: 10.1128/JCM.01798-09

16. Szeto CC, Kam-Tao Li P, Johnson DW, et al . ISPD Catheter-Related Infection Recommendations: 2017 Update. Perit Dial Int. 2017;37(2):141-154. doi: 10.3747/pdi.2016.00120

17. Wu H, Li I, Weng C, et a l. Prophylactic antibiotics for endoscopy-associated peritonitis in peritoneal dialysis patients. PLoS One. 2013;8(8):e71532. doi: 10.1371/journal.pone.0071532

Ethical aspects 
The patient provided written informed consent to the publication of her case. Anonymity has been preserved. Funding

None

Conflict of Interest

None

Author Contributions

Fosso C: followed the case and reviewed the manuscript

Maillart E: reviewed the manuscript

Beun A: followed the case and reviewed the manuscript

Touzani F: followed the case and reviewed the manuscript

Mahadeb B: identified the germ and reviewed the manuscript

Clevenbergh P: followed the case and organized the case report 